Check for updates

Cite this: RSC Adv., 2019, 9, 37598

\title{
Conical microstructuring of titanium by reactive gas assisted laser texturing $\dagger$
}

\author{
Karl Wöbbeking, ${ }^{a}$ Mingji Li, ${ }^{a}$ Eike G. Hübner (D)*ab and Wolfgang Schade ${ }^{a c}$
}

Femtosecond laser micromachining is an important and flexible method to generate precisely targeted surfaces on various materials. On titanium, the laser structuring process strongly depends on the laser parameters. For example, an increasement of the pulse length and repetition rate favors melting processes instead of ablation and microstructuring. We report on an investigation of reactive halogens (iodine, bromine, chlorine) and halocarbons as additives to the laser structuring process of pure titanium and the common alloy Ti-6Al-4V with 0.75 ps laser pulses. The choice of the halogen allows control of whether solely the chemical composition or the surface microstructure should be altered. Bromine was found to be an efficient additive to generate homogeneous microstructures based on micropillars at convenient conditions (air, atmospheric pressure). The resulting surfaces have been characterised by scanning electron microscopy, energy dispersive X-ray spectroscopy, thermal emission infrared photography, reflective UV/Vis spectroscopy and contact angle measurements. The bromine/air processed titanium surfaces revealed superhydrophilicity, strongly increased thermal emissivity and a high absorptivity ("black metal").

Received 30th July 2019

Accepted 12th November 2019

DOI: 10.1039/c9ra05918k

rsc.li/rsc-advances a purely physical process, ${ }^{22}$ i.e. phase explosion and evaporation of the metal by the ultrafast heating process. ${ }^{1}$ A major impact of the processing parameters and the laser setup concerning pulse length and repetition rate on the structuring processes is observed. The influence of the pulse length on the laser structuring process has been analysed for various materials in the literature. ${ }^{23-26}$ In general, the ablation threshold increases with increasing pulse duration..$^{23,25,26}$ Furthermore, melting processes become more important on a longer timescale. ${ }^{24}$ For visualization, a short comparison of material structuring on different laser setups (see Table ST1 in the ESI $\dagger$ for detailed laser parameters) is given in Fig. 1. On aluminium, treatment with $60 \mathrm{fs}$ pulses $\left(J=2.61 \mathrm{~J} \mathrm{~cm}^{-2}, N_{\text {spot }}\right.$ $=250, f=10 \mathrm{kHz}, \mathrm{N}_{2}$ atmosphere) leads to the well-known conical structuring (Fig. 1a). Treatment with comparable parameters but at 0.75 ps pulse length $\left(J=2.61 \mathrm{~J} \mathrm{~cm}^{-2}, N_{\text {spot }}\right.$ $=250, f=1 \mathrm{MHz}, \mathrm{N}_{2}$ atmosphere) only leads to a very weakly structured surface which shows some molten droplets (Fig. 1b). It must be noted, that the repetition rate is strongly increased in the setup with 0.75 ps pulses. The increased scanner speed compensates to the same number of pulses per spot of $N=250$ but heat accumulation can occur due to the fast repetition on each spot.

On some materials such as aluminium and silicon reactive species from ambient gases play an important role and can assist the structuring process. ${ }^{\mathbf{8 , 9}, 22}$ Processing the aluminium sample with the same laser parameters with 0.75 ps pulses, but at air $\left(J=2.61 \mathrm{~J} \mathrm{~cm}^{-2}, N_{\text {spot }}=250, f=1 \mathrm{MHz}\right.$, air) leads to deep craters and oxidation processes strongly differing from the
Stollen 19H, DE-38640 Goslar, Germany

${ }^{b}$ Clausthal University of Technology, Institute of Organic Chemistry, Leibnizstr. 6, DE-38678 Clausthal-Zellerfeld, Germany.E-mail: eike.huebner@tu-clausthal.de

${ }^{c}$ Clausthal University of Technology, Institute of Energy Research and Physical Technologies, Am Stollen 19B, DE-38640 Goslar, Germany

$\dagger$ Electronic supplementary information (ESI) available: All laser parameters, further SEM images and spectra. See DOI: 10.1039/c9ra05918k 
a)

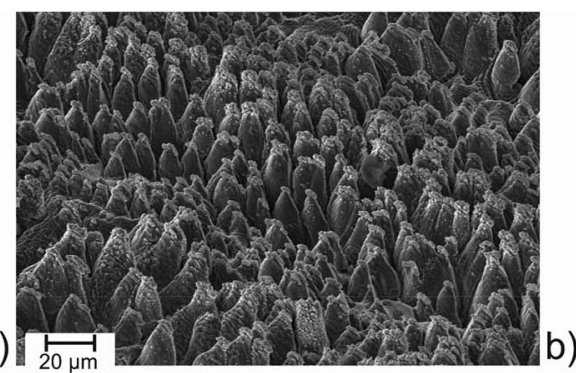

d)

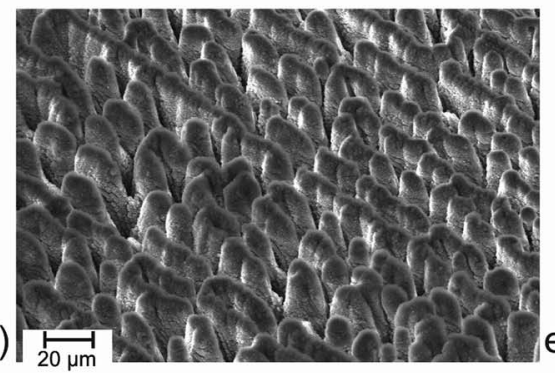

g)!

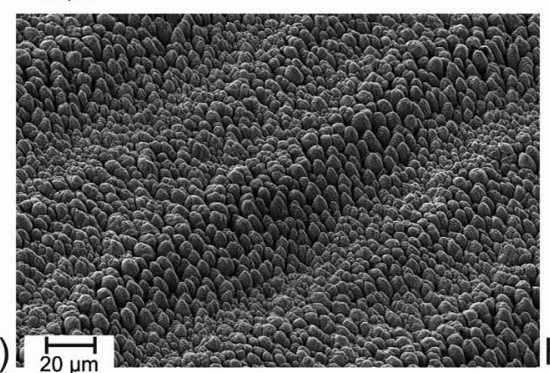

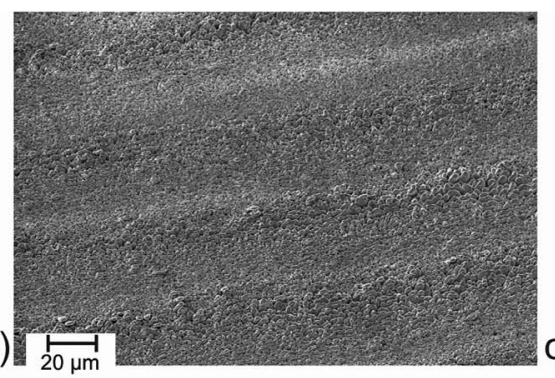
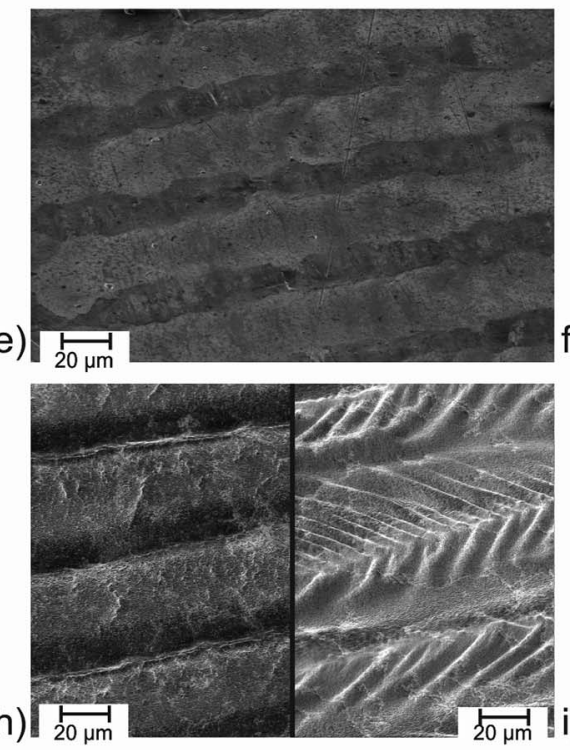
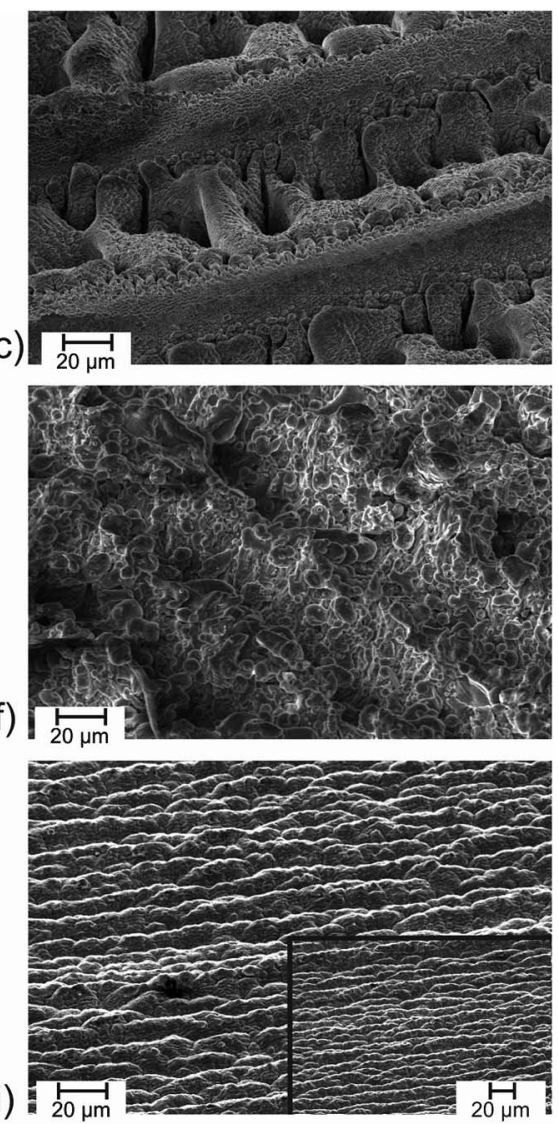

Fig. 1 Laser structuring of aluminium with the following laser parameters: (a) $\lambda=800 \mathrm{~nm}, \tau=60 \mathrm{fs}, \mathrm{J}=2.61 \mathrm{~J} \mathrm{~cm}^{-2}, N_{\text {spot }}=250, f=10 \mathrm{kHz}, \mathrm{N}_{2}$, (b) $\lambda=1030 \mathrm{~nm}, \tau=0.75 \mathrm{ps}, J=2.61 \mathrm{~J} \mathrm{~cm}^{-2}, N_{\text {spot }}=250, f=1 \mathrm{MHz}, \mathrm{N}_{2}$, (c) $\lambda=1030 \mathrm{~nm}, \tau=0.75 \mathrm{ps}, J=2.61 \mathrm{~J} \mathrm{~cm}^{-2}, N_{\text {spot }}=250, f=1 \mathrm{MHz}$, air, (d) $\lambda=1030 \mathrm{~nm}, \tau=0.75 \mathrm{ps}, \mathrm{J}=0.15 \mathrm{~J} \mathrm{~cm}^{-2}, N_{\text {spot }}=1960, f=1 \mathrm{MHz}$, air, (e) $\lambda=1064 \mathrm{~nm}, \tau \approx 2 \mathrm{~ns}, J=3.18 \mathrm{~J} \mathrm{~cm}^{-2}, N_{\text {spot }}=80, f=80 \mathrm{kHz}$, air and (f) $\lambda=1064 \mathrm{~nm}, \tau \approx 2 \mathrm{~ns}, J=12.73 \mathrm{~J} \mathrm{~cm}^{-2}, N_{\mathrm{spot}}=20, f=20 \mathrm{kHz}$, air as well as titanium with the following laser parameters: (g) $\lambda=800 \mathrm{~nm}, \tau=$ $60 \mathrm{fs}, J=1.44 \mathrm{~J} \mathrm{~cm}^{-2}, N_{\text {spot }}=250, f=10 \mathrm{kHz}, \mathrm{N}_{2}$, (h) $\lambda=1030 \mathrm{~nm}, \tau=0.75 \mathrm{ps}, J=1.44 \mathrm{~J} \mathrm{~cm}^{-2}, N_{\text {spot }}=250, f=1 \mathrm{MHz}$, air (left) and $\lambda=1030 \mathrm{~nm}$, $\tau=0.75 \mathrm{ps}, \mathrm{J}=1.44 \mathrm{~J} \mathrm{~cm}^{-2}, N_{\text {spot }}=250, f=1 \mathrm{MHz}, \mathrm{N}_{2}$ (right) and (i) $\lambda=1030 \mathrm{~nm}, \tau=0.75 \mathrm{ps}, J=0.14 \mathrm{~J} \mathrm{~cm}^{-2}, N_{\text {spot }}=942, f=1 \mathrm{MHz}$, air.

sample processed under nitrogen (Fig. 1c). With a reduced fluence and an increased number of hits per spot $(J=0.15 \mathrm{~J}$ $\mathrm{cm}^{-2}, N_{\text {spot }}=1960, f=1 \mathrm{MHz}$, air), a nicely structured aluminium surface can be achieved with 0.75 ps pulses at air (Fig. 1d). The repeated treatment of a surface with several laser shots has a strong impact on the surface structure. ${ }^{27,28}$ Conical microstructures and larger pillars require a number of shots from $N=300$ with the structures becoming more and more dominant with increasing numbers of hits. ${ }^{28}$ The high number of pulses per spot of $N=1960$ allows to compensate the low laser fluence here. Additionally, the line distance during the structuring process was smaller than the spot diameter of the focused laser beam leading to additional sets of pulses on each spot of the surface.

Switching to nanosecond pulses of approx. $2 \mathrm{~ns}(J=3.18 \mathrm{~J}$ $\mathrm{cm}^{-2}, N_{\text {spot }}=80, f=80 \mathrm{kHz}$, air) demonstrates that at this strongly elongated pulse width the surface remains nearly unchanged at a comparable laser fluence (Fig. 1e) while increasing the laser fluence $\left(J=12.73 \mathrm{~J} \mathrm{~cm}^{-2}, N_{\text {spot }}=20, f=20\right.$ $\mathrm{kHz}$, air) led to a mixture of melting and structuring (Fig. 1f).

On titanium, femtosecond laser pulses are widely known to form microconical structures. ${ }^{22,27}$ This is demonstrated in Fig. $1 \mathrm{~g}$ with 60 fs pulses $\left(J=1.44 \mathrm{~J} \mathrm{~cm}^{-2}, N_{\text {spot }}=250, f=10 \mathrm{kHz}, \mathrm{N}_{2}\right)$ in agreement with microcones described in literature obtained with 130 fs pulses. ${ }^{27}$ Basically the same parameters $\left(J=1.44 \mathrm{~J} \mathrm{~cm}^{-2}\right.$, $N_{\text {spot }}=250, f=1 \mathrm{MHz}$ ) with $0.75 \mathrm{ps}$ pulses at $1 \mathrm{MHz}$ only led to long molten trenches instead of microcones. The influence of oxygen is much less pronounced. At air and under an $\mathrm{N}_{2}$ atmosphere comparable surfaces are obtained in these atmospheres (Fig. 1h). To prevent melting processes, the laser fluence was reduced. The influence of the laser fluence on the surface structuring of titanium with femtosecond lasers is wellinvestigated in literature..$^{27,29-31}$ On titanium, a fluence of $J=$ $0.5-1.2 \mathrm{~J} \mathrm{~cm}^{-2}$ (at $130 \mathrm{fs}$ ) is reported to form well-defined conical structures. The size of the microcones increases with the laser fluence. ${ }^{27}$ Here, the laser fluence was reduced to $J=0.14 \mathrm{~J} \mathrm{~cm}^{-2}$ with 0.75 ps pulses at $1 \mathrm{MHz}$ repetition rate to prevent melting. As discussed for aluminium, the low fluence was compensated with a higher number of laser pulses per spot on the surface. Still, under a nitrogen atmosphere the resulting structures $(J=0.14 \mathrm{~J}$ $\mathrm{cm}^{-2}, N_{\text {spot }}=942, f=1 \mathrm{MHz}, \mathrm{N}_{2}$ atmosphere) are weak (see Fig. S1 of the ESI $\dagger$ ). At air, structuring is slightly enhanced $(J=$ $0.14 \mathrm{~J} \mathrm{~cm}^{-2}, N_{\text {spot }}=942, f=1 \mathrm{MHz}$, air), which is in accordance with literature,,$^{22,32,33}$ but conical microstructuring is absent 
(Fig. 1i). The surface shows some similarity with the structure achieved with $130 \mathrm{fs}$ pulses at a low laser fluence. ${ }^{27}$

The ablation threshold for titanium is given in literature with $J=0.10 \mathrm{~J} \mathrm{~cm}^{-2} \cdot{ }^{34}$ Additionally, the fast repetition rate can lead to some accumulative effects. $^{34,35}$ The documented threshold of $J=0.10 \mathrm{~J} \mathrm{~cm}^{-2}$ is a multi-shot threshold based on a repetition rate of $0.1 \mathrm{kHz}$ and incubative effects can lower the threshold. ${ }^{34}$ In general, this effect is rather small on metals, i.e. single-shot and multi-shot threshold are rather close. ${ }^{34,35}$ On the other hand, it has been shown that melting processes of the surface can form a barrier of dielectric material on titanium at fast repetition rates. ${ }^{36}$ Taken together, it can be assumed that the laser fluence of $E=0.14 \mathrm{~J} \mathrm{~cm}^{-2}$ at the repetition rate of 1 $\mathrm{MHz}$ applied here is just right above the ablation threshold which is in agreement with the experimental results of a very weak structuring process.

While the reactive oxygen atmosphere assists in the structuring process of aluminium, these mechanism do not apply for titanium. Examples in literature for the structuring process of silicon have proven the efficiency of other reactive gases on the conical structuring process. ${ }^{\mathbf{8} 9}$ In an $\mathrm{SF}_{6}$ atmosphere, microcones were obtained with fs and ns laser pulses on silicon. ${ }^{9}$ Applying a gas atmosphere of $\mathrm{HCl}$ or $\mathrm{SF}_{6}$ to the femtosecond processing of titanium leads to a suppression of the conical structuring process. ${ }^{22}$

Here, we investigated laser microstructuring of pure titanium and Ti-6Al-4V plates with 0.75 ps laser pulses at air in the additional presence of iodine, bromine and chlorine and organic compounds thereof. Ti-6Al-4V as most used titanium alloy has been chosen as additional substrate due to its high mechanical stability and corrosion resistance. To the best of our knowledge, we report on the first conical structures on titanium prepared with help of highly reactive halogens as additive. Besides the scientific aspect, the extension of the processing parameters for conical microstructured titanium surfaces towards longer laser pulses and high repetition rates typically provided by high-power laser setups is of interest in the view of efficient and cost-effective laser structuring of larger surface areas. ${ }^{37}$

All subsequent experiments concerning various gas atmospheres have been performed with exactly the same laser parameters.

\section{Experimental}

Sheets of titanium (99.6+\%), Ti-6Al-4V and aluminium (99.0\%) (Goodfellow) have been cleaned by ultrasonication with acetone and deionised water. Chemicals have been obtained from Sigma Aldrich unless stated differently. Chlorine (Westfalen) has been dried with conc. $\mathrm{H}_{2} \mathrm{SO}_{4}$. Iodine, bromine, iodoform, chloroform, tetrabromomethane, nitrogen (Linde) and sodium thiosulfate have been used as received. UV/Vis/NIR spectra have been measured with an Uvikon XS spectrophotometer. Scanning electron microscope (SEM) images have been taken with an EVO MA10 (Zeiss) microscope operated at $10 \mathrm{kV}$ with an integrated energy-dispersive X-ray spectroscopy (EDX) unit at 5 and $10 \mathrm{kV}$. A laser scanning microscope LSM 700 (Zeiss) operated at $405 \mathrm{~nm}$ has been used for microscopic depth information. Emissivity measurements have been perfomed at $100{ }^{\circ} \mathrm{C}$ with a Testo T885 IR camera. Surface UV/Vis-spectra have been recorded with a Jasco V650 spectrophotometer equipped with an integrating sphere (Ulbricht sphere Jasco ISV-722). Contact angles have been measured from photographic images of water droplets of $10 \mu \mathrm{L}$ volume on the cleaned samples after $24 \mathrm{~h}$ and $15 \mathrm{~s}$ after application of the drop.

Laser microstructuring has been performed with an AMPHOS 400 Yb:YAG high power laser system at a centre wavelength of $\lambda=1030 \mathrm{~nm}$ and a pulse length of $\tau=0.75 \mathrm{ps}$. A spot diameter of approximately $95 \mu \mathrm{m}\left(1 / e^{2}\right)$ after a $420 \mathrm{~mm} f$ theta objective has been applied. The samples have been processed with a continuous speed of $v=0.1 \mathrm{~m} \mathrm{~s}^{-1}$. The average output power of $P=9.75 \mathrm{~W}$ in combination with the repetition rate of $f=1 \mathrm{MHz}$ results in a pulse energy of $E=9.75 \mu \mathrm{J}$ and a fluence of $J=0.14 \mathrm{~J} \mathrm{~cm}^{-2}$ per shot. Each spot on the surface has been hit $N=942$ times by the laser beam. Areas of $10 \times 10$ $\mathrm{mm}^{2}$ have been irradiated in lines with a distance of $D=7 \mu \mathrm{m}$. The general laser setup has been described previously. ${ }^{17}$ For comparison, samples have been processed with a Ti:sapphire laser system with $\tau=60 \mathrm{fs}$ laser pulses at a centre wavelength of $\lambda=800 \mathrm{~nm}$ according to our previously published setup. ${ }^{\mathbf{1 9}}$ Aluminium samples treated with nanosecond laser pulses have been obtained from Trotec.

Laser microstructuring has been processed within an airtight stainless steel chamber (inner diameter $10 \mathrm{~cm}$ ) equipped with inlet and outlet valves and a borosilicate window. The total

Table 1 Physical properties of $\mathrm{Ti}_{1} \mathrm{TiO}_{2}$, and the halogens and halides discussed here

\begin{tabular}{lllll}
\hline & $\mathrm{Mp}^{38}\left({ }^{\circ} \mathrm{C}\right)$ & $\mathrm{Bp}^{38}\left({ }^{\circ} \mathrm{C}\right)$ & Vapor pressure $^{38}$ (mbar) @ 25 ${ }^{\circ} \mathrm{C}$ & Electronegativity $^{38}$ \\
\hline $\mathrm{Ti}$ & 1668 & 3287 & - & - \\
$\mathrm{TiO}_{2}$ & 1843 & $\sim 3000^{a}$ & - & $-5^{b}$ \\
$\mathrm{I}_{2}$ & 114 & 184 & 246 & 2.7 \\
$\mathrm{Br}_{2}$ & -7 & 59 & $>1013$ & 3.0 \\
$\mathrm{Cl}_{2}$ & -102 & -34 & 229 & - \\
$\mathrm{CHCl}_{3}$ & -63 & 61 & $<0.6^{c}$ & - \\
$\mathrm{TiI}_{4}$ & 150 & 230 & 13 & - \\
$\mathrm{TiBr}_{4}$ & 39 & 137 & & - \\
$\mathrm{TiCl}_{4}$ & -24 & &
\end{tabular}


volume of the chamber is $0.65 \mathrm{~L}$. The chamber has been evacuated to be refilled with gases or equilibrated with the vapor pressure of solid substances in vacuo and refilled with air or equilibrated with the vapor pressure of liquids at air.

Samples have been cleaned by ultrasonication in deionised water for $5 \mathrm{~min}$. Samples have been stored at ambient conditions in the dark. The chamber has been washed with sodium thiosulfate solution after each process to remove residual halogens.

\section{Discussion}

The laser setup used here is a high-power Yb:YAG laser system at $\lambda=1030 \mathrm{~nm}$ suitable for continuous operation and high radiation output operated with $0.75 \mathrm{ps}$ pulses and at a repetition rate of $1 \mathrm{MHz}$ as typical parameters for industrial laser systems.

To assist the surface ablation process of titanium, reactive halogens were added to the processing chamber. The basic idea is the fast formation of titanium halides due to the high reactivity of halogens and rapid evaporation of the reaction products due to their low boiling point (see Table 1 and Fig. 2).

The resulting $\mathrm{Ti}-\mathrm{X}(\mathrm{X}=\mathrm{I}, \mathrm{Br}, \mathrm{Cl})$ terminated surface should be transformed into a titanium oxide/ $\mathrm{Ti}-\mathrm{OH}$ terminated surface immediately after contact with ambient air/water due to the high reactivity of the titanium halides (Fig. 2). We applied an inert processing chamber made of stainless steel and a borosilicate window to handle the reactive gas atmosphere (Fig. 3ac). To cross-check the transmissibility of the intensively colored gas atmosphere at the laser wavelength of $1030 \mathrm{~nm}, \mathrm{UV} / \mathrm{Vis} / \mathrm{NIR}$ (near infrared) spectra of the gases were recorded (Fig. 3d) and prove complete NIR transparency.

On titanium and Ti-6Al-4V plates, squares of $1 \mathrm{~cm} \times 1 \mathrm{~cm}$ have been processed in the chamber. Assuming a maximal increase of the surface area by the structuring process by a factor of 60 (taken from BET measurements of structured titanium plates with a 60 fs laser setup ${ }^{19}$ ) and a surface density of terminal M-OH (M-X) groups of $6 \mathrm{~nm}^{-2}, 403 \times 10^{-5} \mathrm{mmol}$ of reagent could be consumed during the process. Considering the volume of the chamber $(0.65 \mathrm{~L})$, this is achieved at a vapor pressure of 0.001 mbar, which is several magnitudes below the substances investigated here (Table 1). Each structuring process has been repeated on the same material without opening the chamber to prove that consumption of the reactive gas was not a limiting factor.

It must be noted, that some synergetic effects can be expected comparing the halogens as additive. The reactivity

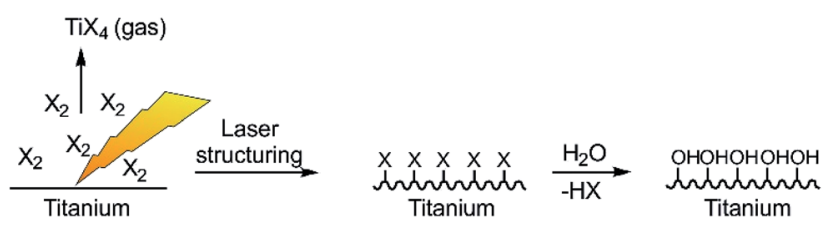

Fig. 2 Sketch of the laser structuring process in presence of reactive halogens and formation of low boiling titanium halides as well as the reactive titanium surface.

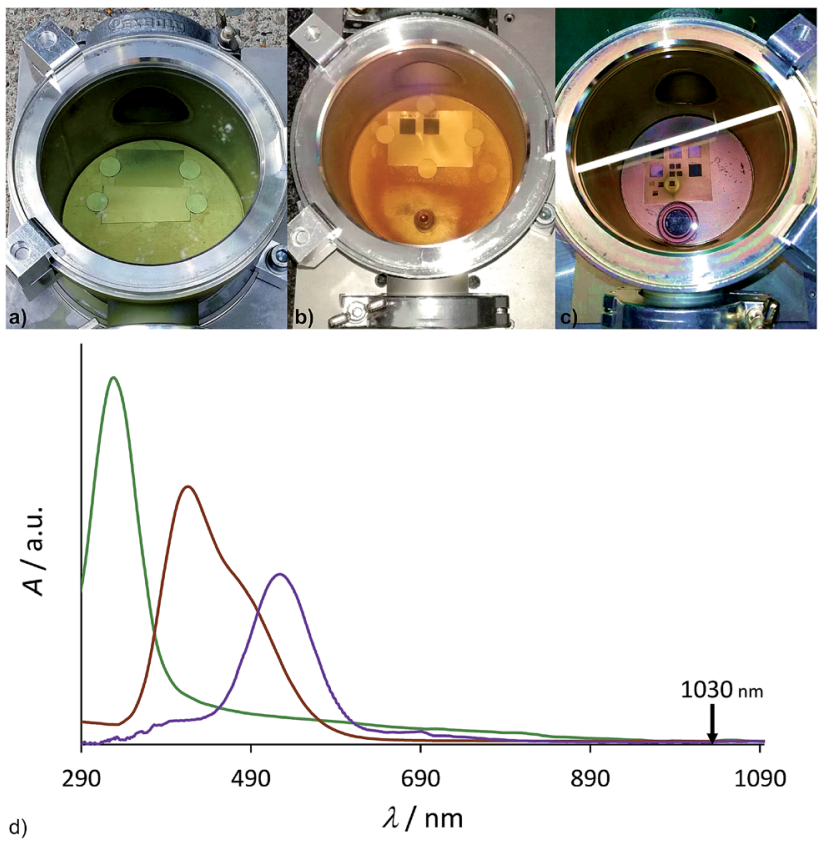

Fig. 3 Processing chamber flooded with mixtures of air and (a) chlorine, (b) bromine and (c) iodine and (d) UV/Vis/NIR spectra of the gases (green: chlorine, brown: bromine, purple: iodine) and incident laser wavelength.

(roughly estimated by the electronegativity) increases from iodine to chlorine, the vapor pressure (i.e. the concentration inside the chamber) increases, too, and the boiling point of the resulting titanium halide decreases from $\mathrm{TiI}_{4}$ to $\mathrm{TiCl}_{4}$, facilitating the evaporation of the product (see Table 1).

First, iodine was investigated as additive. The impact on the structure formation was found to be negligible. The SEM images reveal a similar structure at air with (Fig. 4b) and without (Fig. 1i) additional iodine. The surface composition was determined by energy-dispersive X-ray spectroscopy (EDX) and reveals the complete absence of residual iodine on the surface (Table 2). Furthermore, an unchanged oxygen content of the oxide layer on the $\mathrm{Ti} / \mathrm{Ti}-6 \mathrm{Al}-4 \mathrm{~V}$ plates produced during the laser process at air with and without iodine allows concluding, that the physical (SEM) and chemical (EDX) parameters of the surface are identical. Consequently, the surface parameters investigated here, i.e. the water contact angle, the absorptivity in the UV/Vis region and the thermal emissivity at $100{ }^{\circ} \mathrm{C}$ are identical, too (Table 2). To check if a higher fraction of iodine in the surrounding atmosphere does have an impact on the surface structuring process, the experiments were repeated at a reduced pressure. At 3 mbar (i.e. 0.6 mbar $\mathrm{O}_{2}$ in air), the atmosphere should consist of a mixture of oxygen and iodine (v.p. 0.5 mbar) at roughly comparable fractions. The structuring of the titanium surface under vacuum differs significantly from structuring at air since evaporation processes are enforced. Femtosecond laser structuring of titanium under low pressure conditions is well investigated in literature ${ }^{22,31,41}$ and can lead to very well defined microcones with 130 fs laser pulses. ${ }^{22}$ With $0.75 \mathrm{ps}$ pulses at a repetition rate of $1 \mathrm{MHz}$, we found the surface 
a)

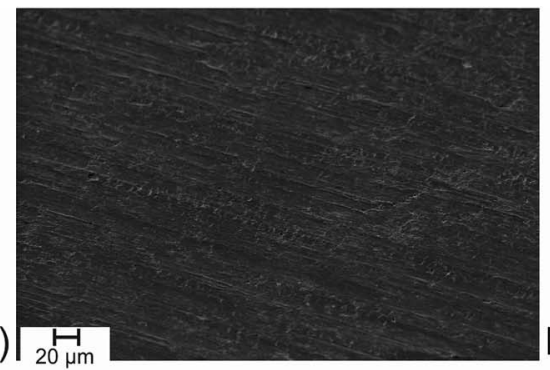

d)

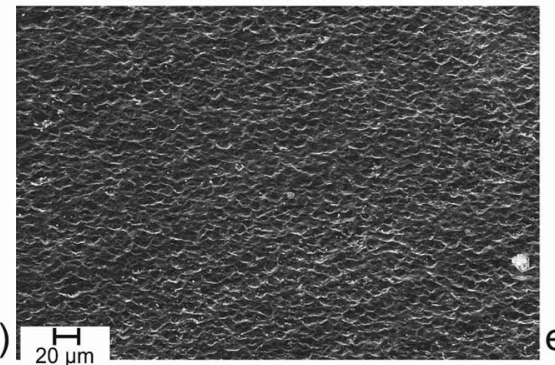

b)|
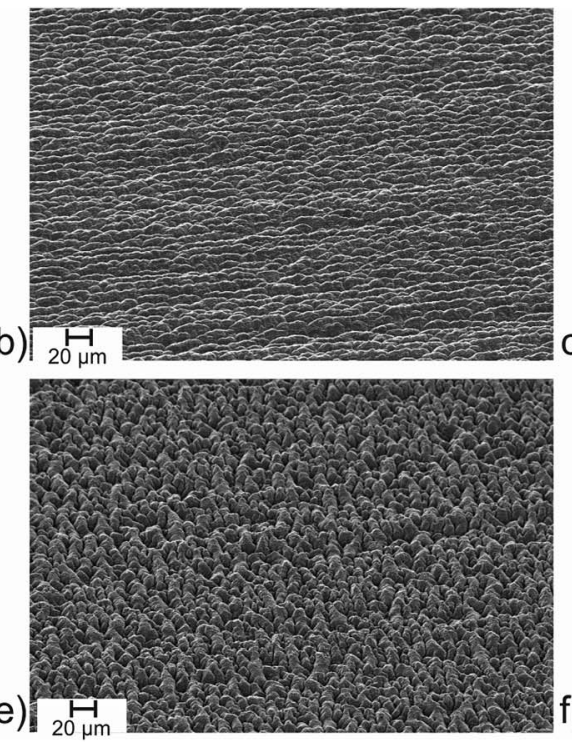
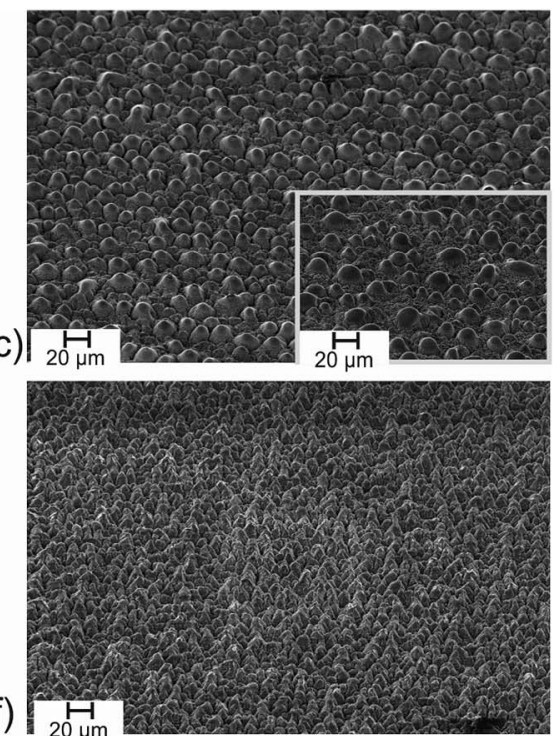

Fig. 4 SEM images of (a) neat titanium surface and laser processed in (b) air/iodine, (c) air/bromine (inset: Ti-6Al-4V), (d) air/chlorine, (e) vacuum and (f) vacuum/iodine atmosphere. The laser parameters are similar to the sample processed at air (Fig. 1i): $\lambda=1030 \mathrm{~nm}, \tau=0.75 \mathrm{ps}, \mathrm{J}=0.14 \mathrm{~J}$ $\mathrm{cm}^{-2}, N_{\text {spot }}=942, f=1 \mathrm{MHz}$ in all cases.

to consist of irregular microchannels (Fig. 4e), a common structure in laser structuring and occasionally referred to as chaotic structure. Obviously, structuring processes are much more pronounced compared to atmospheric pressure, but the formation of microcones was not observed. Again, the presence of iodine did not change the surface structuring process and the SEM images with and without iodine are nearly identical (Fig. 4f). As an interesting aspect, the contact angle of the structure processed at iodine/vacuum conditions revealed a superhydrophilic surface. Although the contact angle of the structured surface achieved in vacuum is decreased in comparison to the sample processed in air (Table 2), the hydrophilicity is significantly enhanced further by the iodine atmosphere. This can be explained by the mechanism shown in Fig. 2. While in vacuum a pure metal surface is formed, which builds up a thin oxide layer on top, the presence of iodine leads to a Ti-I terminated surface. Once the chamber is opened and the surface gets into contact with humid air, and finally the cleaning step with deionised water, transforms the surface into an Ti-OH terminated, superhydrophilic surface. Enhancing the hydrophilicity of laser structured titanium oxide surfaces up to contact angles close to zero by subsequent chemical treatment

Table 2 Properties of the Ti and Ti-6Al-4V plates treated with laser pulses in various atmospheres

\begin{tabular}{|c|c|c|c|c|c|c|c|}
\hline \multirow[b]{2}{*}{ Sample } & \multirow[b]{2}{*}{ Condition } & \multicolumn{2}{|c|}{$\begin{array}{l}\text { Surface composition } \\
\text { (atom\%) }\end{array}$} & \multirow[b]{2}{*}{ Contact angle $\left(^{\circ}\right)$} & \multirow[b]{2}{*}{ Emissivity $^{e}(\%)$} & \multirow[b]{2}{*}{ Absorptivity ${ }^{f}(\%)$} & \multirow[b]{2}{*}{ Surface Roughness ${ }^{g} R_{\mathrm{a}}(\mu \mathrm{m})$} \\
\hline & & $\mathrm{X}(=\mathrm{Cl}, \mathrm{Br}, \mathrm{I})$ & $\mathrm{O}$ & & & & \\
\hline $\mathrm{Ti}$ & Untreated & - & $19.5 \pm 1.2$ & $45 \pm 3$ & $21 \pm 1$ & 67 & 0.34 \\
\hline TiAlV & Untreated & - & $17.4 \pm 2.0$ & $32 \pm 3$ & $20 \pm 1$ & 67 & 0.58 \\
\hline Ti-ref $^{a}$ & $\mathrm{~N}_{2}^{a}$ & - & $12.4 \pm 0.7^{c}$ & $\rightarrow 0^{d}$ & $67 \pm 2$ & 98 & 1.88 \\
\hline Ti-air & Air & - & $63.2 \pm 3.7$ & $55 \pm 3$ & $46 \pm 4$ & 95 & 1.56 \\
\hline TiAlV-air & Air & - & $50.8 \pm 2.4$ & $58 \pm 3$ & $54 \pm 2$ & 96 & 1.69 \\
\hline $\mathrm{Ti}-\mathrm{I}_{2}$ & Air $+\mathrm{I}_{2}$ & 0.0 & $65.8 \pm 3.0$ & $54 \pm 3$ & $47 \pm 2$ & 95 & 1.12 \\
\hline TiAlV-I $_{2}$ & Air $+\mathrm{I}_{2}$ & 0.0 & $50.2 \pm 2.3$ & $56 \pm 3$ & $54 \pm 2$ & 96 & 1.24 \\
\hline Ti-vac & $3 \mathrm{mbar}$ & - & $57.0 \pm 3.1$ & $17 \pm 3$ & - & - & 1.25 \\
\hline Ti-vac- $\mathrm{I}_{2}$ & $3 \mathrm{mbar}+\mathrm{I}_{2}$ & $0.7 \pm 0.05$ & $63.5 \pm 3.6$ & $\rightarrow 0$ & - & - & 1.23 \\
\hline $\mathrm{Ti}-\mathrm{Br}_{2}$ & $\mathrm{Air}+\mathrm{Br}_{2}$ & $0.2 \pm 0.02$ & $71.1 \pm 3.8$ & $\rightarrow 0$ & $75 \pm 5$ & 96 & 2.34 \\
\hline TiAlV- $\mathrm{Br}_{2}$ & Air $+\mathrm{Br}_{2}$ & 0.0 & $68.2 \pm 3.6$ & $\rightarrow 0$ & $71 \pm 5$ & 97 & 2.55 \\
\hline TiAlV- $\mathrm{Cl}_{2}$ & $\mathrm{Air}+\mathrm{Cl}_{2}^{b}$ & $0.4 \pm 0.03$ & $16.0 \pm 1.4$ & $34 \pm 3$ & $24 \pm 4$ & 59 & 0.74 \\
\hline $\mathrm{Ti}-\mathrm{CHCl}_{3}$ & $\mathrm{Air}+\mathrm{CHCl}_{3}$ & $<0.1$ & $63.9 \pm 3.9$ & $66 \pm 3$ & $56 \pm 9$ & 82 & 0.78 \\
\hline TiAlV-CHCl ${ }_{3}$ & $\mathrm{Air}+\mathrm{CHCl}_{3}$ & $<0.1$ & $63.1 \pm 3.7$ & $72 \pm 3$ & $55 \pm 8$ & 87 & 0.57 \\
\hline
\end{tabular}

${ }^{a}$ Processed on a Ti:sapphire laser setup under $\mathrm{N}_{2}$ for comparison (see Fig. $1 \mathrm{~g}$ ). ${ }^{b} 600 \mathrm{mbar}$ air/400 $\mathrm{mbar} \mathrm{Cl}_{2} \cdot{ }^{c} \mathrm{~N}_{2}: 16.1 \% .{ }^{d}$ Increases to $>1400^{\circ}$ after some days. ${ }^{e}$ Measured at $100{ }^{\circ} \mathrm{C} .{ }^{f}$ At $600 \mathrm{~nm}$; calcd from diffuse reflectance spectra. ${ }^{g}$ From LSM. 
with reagents such as $\mathrm{NaOH}$ and $\mathrm{H}_{2} \mathrm{O}_{2},{ }^{2}$ commonly used to hydroxylate metal oxidic structures leading to a high amount of terminal $\mathrm{M}-\mathrm{OH}$ groups, is a well-known effect. ${ }^{40,42,43}$ The assumption of an hydroxylic surface caused by the iodine-rich processing conditions is backed by the EDX analysis of the surface revealing a low residual amount of iodine ( $<1$ atom\%) but a strong increase of the oxygen content by 6.5 percentage points (Table 2). Compared to results given in literature for laser-processed titanium samples under high-vacuum conditions, even the surface processed under vacuum here is oxygenrich. ${ }^{41}$ This is readily explained by the pressure of 3 mbar applied here providing sufficient oxygen to form $\mathrm{TiO}_{2}$.

Due to the high reactivity of the halogen containing surface we were not able to gather EDX data from the surfaces before hydrolysis. Unfortunately, we observed a strong contamination of the processing chamber with titanium dust during the laser structuring process under all vacuum conditions. We were not able to process areas larger than $16 \mathrm{~mm}^{2}$ for these samples. Hence we could not collect reliable emission and absorption spectra for the vacuum processed samples.

Moving to chlorine as the highest reactive halogen as additive showed a strong impact on the laser structuring process. A chlorine partial pressure of 400 mbar $(+600$ mbar air) was applied to be roughly comparable to bromine (see below). The plate of pure titanium was damaged by the laser impact and deep craters destroyed the sample. The more resistant Ti-6Al-4V sample showed intense surface ablation, too. The resulting surface is rather smooth according to the SEM image (Fig. 4d) and has a silvery finish (Fig. 5a). The step caused by the laser ablation is visible by eye (Fig. 5a) and by laser scanning microscopy (LSM) a depth of $30 \mu \mathrm{m}$ was determined for the etched layer (Fig. 5b). The smooth surface provided surface properties rather close to the untreated material. The thermal emissivity at $100^{\circ} \mathrm{C}$ (measured by IR photography) of $\varepsilon=24 \%$ is the usual emissivity of a titanium metal surface and the UV/Vis reflectance spectra (see Fig. S6 of the ESI†े) confirm the visual appearance of the reflective titanium surface. The reflectivity is slightly increased in comparison to the initial surface (see Table 2). The surface composition does not reveal significant amounts of residual chlorine and does not show an increase of the oxygen content. This can be explained by the fast reaction of chlorine with Ti leading to evaporation of the resulting $\mathrm{TiCl}_{4}$ which prevents the formation of porous oxidic structures. Furthermore, highly reactive $\mathrm{Ti}-\mathrm{Cl}$ residues on the surface can

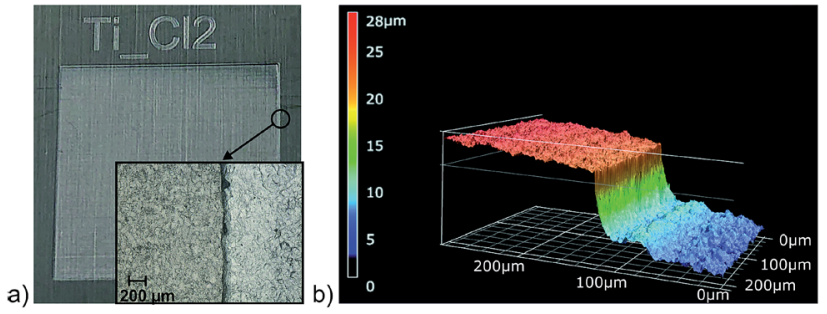

Fig. 5 Plate of Ti-6Al-4V laser processed in a chlorine/air atmosphere. (a) Photographic and microscopic image of the plate and (b) depth information from LSM. undergo condensation with adjacent $\mathrm{Ti}-\mathrm{OH}$ groups leading to $\mathrm{Ti}-\mathrm{O}-\mathrm{Ti}$ structures just as in dehydroxylated surfaces. Overall, we conclude that chlorine is too reactive to assist in structure generating processes even at a reduced partial pressure of 400 mbar, but it leads to a strong enhancement of the laser ablation. Finally, bromine was investigated as medium-reactive halogen. The vapor pressure (Table 1) is high enough to provide a concentration of reactive species comparable to the chlorine/ air mixture. The titanium sample shows a very pronounced structure after processing (Fig. 4c). The surface is homogeneously covered with pillars. At a first look, the surface is rather comparable to the surface obtained with $60 \mathrm{fs}$ pulses at inert conditions (Fig. 1g), although it must be remarked that the pillars are thicker and the structure size is coarser. Additionally, the pillars seem to be cropped and do not provide sharp conical spikes, but a round cap. Pure titanium and Ti-6Al-4V provide nearly the same structure after processing (Fig. $4 \mathrm{c}$ and inset). The homogeneous appearance of the structure is confirmed by the visible inspection of the sample (Fig. 6a) which has a uniformly dark black appearance. The laser structuring process worked well in the same chamber for subsequent squares, proving the process to be routinely usable for larger areas.

The dark black appearance of the surface is typical for conical structures. Of course, it has to be noted that already the air-structured sample shows strong light absorption in the visible region (Fig. 6f) caused by the porous surface structure. Still, the bromine/air processed samples are of deeper colour, which is clearly visible in the reflective UV/Vis spectra (Fig. 6f). The reference sample processed on a Ti:sapphire setup (see Fig. 1g) does show a further increased absorptivity (Fig. 6f). This can be explained by smaller conical structures which can act as a light trap more efficiently. The absorptivity values $\alpha$ given in Table 1 are recalculated from the reflective UV/Vis spectra and reveal an absorptivity of 96-97\% (at $600 \mathrm{~nm}$ ) for the bromine/air processed samples. A simple $A=\log \left(R^{-1}\right)$ conversion has been used for the estimation of $\alpha$, using more sophisticated methods such as Kubelka-Munk transformation ${ }^{44}$ reveals even lower reflectivities, consequently the values given in Table 1 can be considered as the lower limit of the absorptivity. The thermal emissivity of the bromine/air processed samples is strongly increased (Fig. 6c). Emissivity increasement of femtosecondlaser processed structures is well-known in literature. ${ }^{10}$ The air-processed sample shows an increasement of $\varepsilon$ from $20 \%$ (plane titanium surface) to $46 \%$. The reference microconical sample provides an emissivity of $67 \%$ and the bromine/air processed sample reaches $\varepsilon=75 \%$ (Table 2 and Fig. 6c). Again, the results are nearly identical for pure titanium and Ti$6 \mathrm{Al}-4 \mathrm{~V}$.

The contact angle of the bromine/air processed samples reaches down to zero (Fig. 6d). In fact, the water drop is immediately spread on the laser-structured square and finally limited by the borders of the structured surface. The explanation of the superhydrophilicity is rather straightforward having in mind the discussion of the vacuum/iodine samples given above. In this case, the bromine concentration in air is high enough to lead to the same effect as iodine in the vacuum 
a)
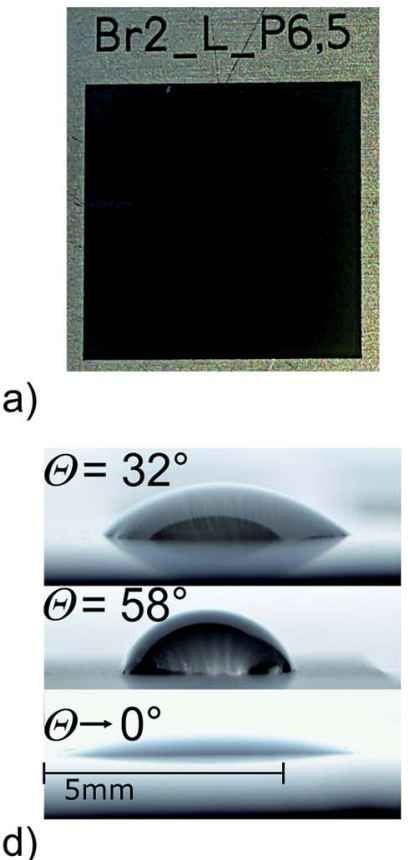

b)
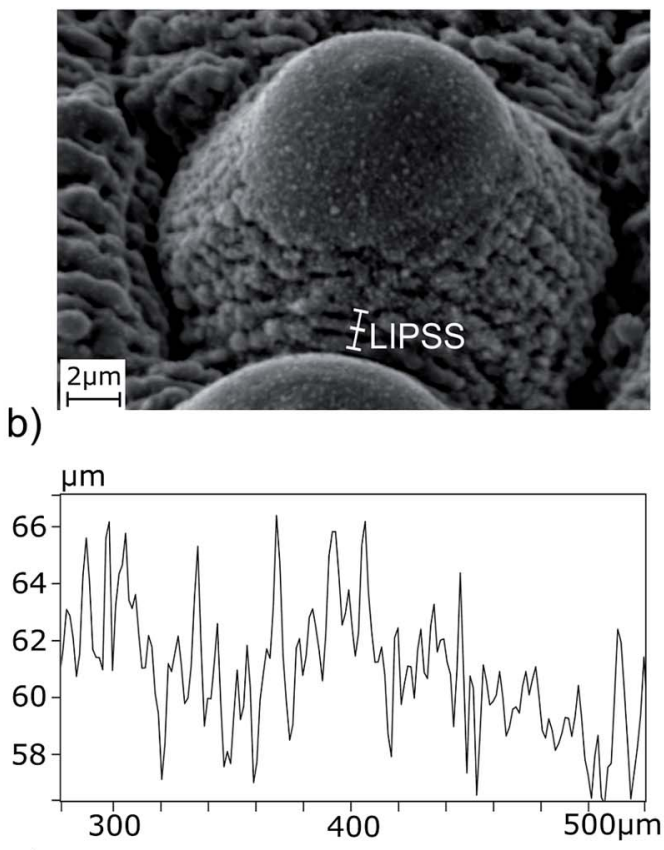

e)

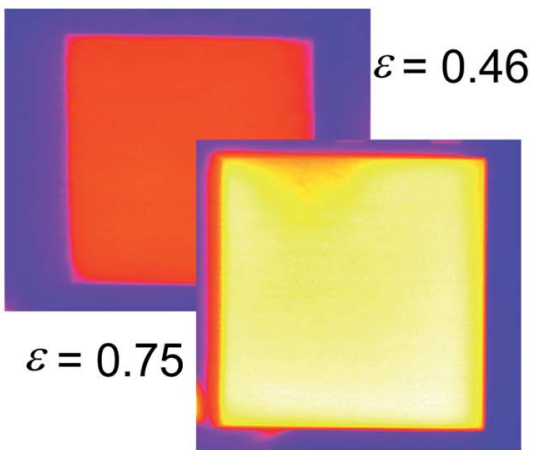

c)

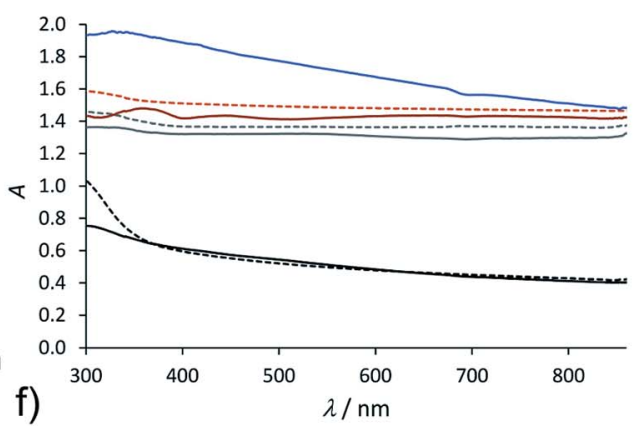

Fig. 6 Titanium sample laser processed in a bromine/air atmosphere. (a) Photographic image, (b) close-up view of the pillar (SEM), (c) thermal emission MIR image at $100^{\circ} \mathrm{C}$ of the air processed sample (top left) and bromine/air processed sample (bottom right) (surrounding area: plane titanium), (d) water contact angle on plane Ti-6Al-4V (top), air-processed sample (middle) and bromine/air processed sample (bottom), (e) height profile of the structured Ti surface and (f) reflective UV/Vis spectra of pure titanium (solid lines) and Ti-6Al-4V (dashed lines) untreated (black), processed at air (grey), processed at bromine/air (brown) and processed on a Ti:sapphire laser setup (see Fig. 1g) for reference (blue).

atmosphere and leads to a Ti-Br terminated surface which is immediately hydrolysed upon contact with water. The discussion is again backed by the results of the EDX analysis which reveals an increased oxygen content for the bromine/air processed titanium sample of 71 atom\% O (compared to 63 atom\% $\mathrm{O}$ processed at air). These results match quite nicely with a rough estimation of a titanium oxide structure $\left(\mathrm{TiO}_{2}: 66\right.$ atom\% $\mathrm{O}$ ) in comparison to a completely $\mathrm{Ti}-\mathrm{OH}$ terminated surface (" $\mathrm{TiO}_{2.5} \mathrm{H}$ ": 71 atom\% $\mathrm{O}$ ). Fortunately, the bromine content is very low (Ti: 0.2 atom $\% \mathrm{Br}$, Ti-6Al-4V: 0.0 atom\% $\mathrm{Br}$ ) and at the edge of the detection limit. Consequently, the surfaces can be expected to be basically halogen free. Comparing the chemical surface composition from the surfaces structured in the presence of iodine, bromine and chlorine, the oxygen-content of the samples increases from the iodine/air atmosphere (at $3 \mathrm{mbar}$ ) to bromine/air and is reduced in the chlorine/air atmosphere. These results match with the observed influence of the additive on the structuring process, which is negligible for iodine, strong for bromine and counteractive for chlorine due to over-etching. The differing Ti-OH density on the surface should lead to an influence on the amount of $\mathrm{H}$-bonded vs. isolated surface $\mathrm{OH}$ groups. ${ }^{40}$ Unfortunately, owed to the surface roughness (Table 2) sufficiently resolved ATR-IR spectra to further analyse the surface $\mathrm{OH}$ groups could not be obtained. In accordance with literature, the superhydrophilicity is lost after several days at air. ${ }^{2}$ In contrast to the reference sample Tiref, which changes its wetting properties rather fast from superhydrophilic to superhydrophobic $\left(\theta>140^{\circ}\right)$ the hydrophilicity on $\mathrm{Ti}-\mathrm{Br}_{2}$ and $\mathrm{TiAlV}-\mathrm{Br}_{2}$ remains longer and the contact angle slowly increases to $60^{\circ}$ within 14 days.

Looking at the microcones closer reveals a significant substructure of the pillars (Fig. 6b). The formation of selforganised microstructures by femtosecond laser structuring is a well-investigated and intensely discussed topic in laser structuring. ${ }^{45-48}$ The laser induced periodic surface structures (LIPSS) are formed perpendicular to the polarization of the laser beam with a periodicity in the order of the laser beam or slightly below. With laser pulses on the picosecond timescale such parallel lines have been identified, too. ${ }^{48}$ At the bottom of the pillars formed here, these oriented microstructures can be identified at a distance of 700-800 $\mathrm{nm}$ matching the expected value for titanium treated with laser pulses at $1030 \mathrm{~nm} .^{46}$ The top of the pillars shows a molten appearance and is not covered with a substructure. The cones itself are not clearly oriented periodically on the surface (Fig. 4c). This isotropic surface structuring results in isotropic surface properties such as the spreading of a water drop which did not show any preferred orientation here although the underlying periodic substructures might affect other parameters. The profile measurement of the surface (Fig. 6e) reveals each pillar to be surrounded by a small crater and height of the pillars is determined to $6-8 \mu \mathrm{m}$, a common value for microcones obtained by femtosecond laser machining. ${ }^{9,13}$ Overall, we conclude bromine to have a strong impact on the structuring process and it is well-suited as additive to generate homogeneous and conically structured surfaces with longer laser pulses. It must be noted, that the 
deposition of titanium from $\mathrm{TiBr}_{4}$ by laser chemical vapor deposition is described in literature and the decomposition of $\mathrm{TiBr}_{4}$ formed during the ablation process here could have an impact on the structuring process. ${ }^{49}$ The influence of reactive gases on the structuring process has been described in literature for silicon. In the presence of $\mathrm{SF}_{6}$ and $\mathrm{Cl}_{2}$, spikes have been obtained with femtosecond and even nanosecond laser pulses. ${ }^{\mathbf{8 9}}$ With longer pulses, coarser and taller structures have been obtained. In contrast to the results observed on titanium here, the addition of $\mathrm{SF}_{6}$ and $\mathrm{Cl}_{2}$ led to sharp spikes while under a nitrogen atmosphere round tips have been formed.

Finally, the application of organic halocarbons as additive to the laser structuring process was investigated. The halocarbons could provide a stable source for halogens at ambient conditions. $\mathrm{SF}_{6}$ is routinely applied as a source for fluorine in femtosecond laser structuring and has shown to prevent the formation of microcones on titanium by over-etching. ${ }^{22}$ The addition of $\mathrm{CBr}_{4}$ and $\mathrm{CHI}_{3}$ to the processing chamber did not show any influence on the laser structuring process at air. This can readily be explained by the low vapor pressure $\left(\mathrm{CBr}_{4}: 1\right.$ mbar, $\mathrm{CHI}_{3}:<0.01 \mathrm{mbar}^{38}$ and is consistent with the results obtained with pure iodine. The application of titanium samples covered with a layer of $\mathrm{CBr}_{4}$ and $\mathrm{CHI}_{3}$ prepared by dip-coating from 10 percent solutions in acetone was not successful, either. The coated layer shielded the underlying titanium surfaces and slow warming of the metal plate led to evaporation of the coated film during the ongoing process. Processing the samples under a layer of liquid chloroform $\left(\mathrm{CHCl}_{3}\right)$ led to intense bubble formation. An unchanged titanium surface under the conditions applied here has been obtained although femtosecond laser processing in aqueous solutions has been reported in literature. ${ }^{\mathbf{5 0}}$

Under a gaseous atmosphere of chloroform/air at a vapor pressure of $\mathrm{CHCl}_{3}$ comparable to bromine (see Table 1), a strong impact on the laser process was observed. Chloroform is totally transparent in the NIR region (see Fig. S5 of the ESI $\dagger$ ) and consequently the laser structuring process proceeded well. The resulting surface showed a homogeneous diffuse greyish appearance (see Fig. S7 of the ESI $\dagger$ ). The unusual optical behaviour was confirmed in the reflective UV/Vis spectra (see Fig. S6 of the ESI $\dagger$ ) which reveal a uniform absorptivity over the full length of the visible spectrum. The absorptivity is calculated to $\alpha=82 \%$ (Table 2 ) which is in accordance with the dull appearance. The thermal emissivity of $\varepsilon=56 \%$ is significantly increased in comparison to the untreated sample (see Fig. S8 of the ESI $\dagger$ ). According to the SEM images (see Fig. S3 and S4 of the ESI $\dagger$ ), the surface has not been structured significantly. The structure is rather flat with some molten droplets and areas. On the other hand, the EDX analysis (Table 2) reveals a high oxygen content. Taking these results together, it may be concluded that a thin $\mathrm{TiO}_{2}$ surface has been formed. The absorption spectra could in this case not be explained by the surface structure, but a potentially oxygen-deficient $\mathrm{TiO}_{2}$ ("black $\mathrm{TiO}_{2}$ ") which is formed at these oxygen-poor conditions. Oxygen-deficient titanium oxides can appear in multiple colors and show strong absorbance in the visible region. ${ }^{51}$ Again, these results could be confirmed on Ti-6Al-4V leading to nearly identical results as on pure titanium. Overall, chloroform is too inert to assist in the surface ablation. Instead, it acts as an inert gas atmosphere with a residual partial pressure of oxygen high enough for oxidation processes of the surface.

\section{Conclusions}

Laser structuring of titanium is strongly affected by the laser parameters such as pulse length and repetition rate of the laser system. In an inert gas atmosphere, at air and in vacuum, conical microstructures are commonly obtained with Ti:sapphire laser setups with femtosecond pulses and repetition rates in the $\mathrm{kHz}$ range. Switching to longer pulse lengths and higher repetition rates typical for high-power laser setups leads to a significant decrease of the surface structuring process. This is in contrast to some other materials, where reactive gases (in the simplest case oxygen from ambient air) assist structure generation and the process is more tolerant. Here, we investigated the addition of halogens to the laser structuring process of titanium with $0.75 \mathrm{ps}$ laser pulses at $1 \mathrm{MHz}$ repetition rate. In dependency of the halogen, the following observations have been made:

- Low reactive iodine does not affect the structuring process. At a reduced pressure, the addition of iodine can change the chemical surface composition.

- Medium reactive bromine has a strong impact on the surface structure. Well-defined microstructures and a surface covered with pillars can be obtained in a bromine/air atmosphere.

- Highly reactive chlorine leads to fast etching of the material during the laser process and smooth surfaces are obtained.

The laser-structuring process in the presence of bromine leads to very homogeneous surfaces. The physical characterization of the surface is in accordance with its conical microstructure. The surfaces provide high thermal emissivity, a high absorptivity and superhydrophilicity. The latter is explained by a change of the chemical composition of the surface. Immediate hydrolysation of the processed samples upon contact with humid air/water results in a halogen-free and oxygen-rich material. In the experiments presented here, $300 \mu \mathrm{L}$ of bromine are sufficient to saturate the processing chamber and several samples could be processed in a row within the same atmosphere.

\section{Conflicts of interest}

There are no conflicts to declare.

\section{Acknowledgements}

We gratefully acknowledge financial support from the German Federal Ministry for Economic Affairs and Energy (BMWi) within the context of the project GreenH2 (contract 03ET6058A) and from the Open Access Publishing Fund of the Clausthal University of Technology. We sincerely thank Andreas Gabler (Fraunhofer Heinrich Hertz Institute) and Thomas Gimpel (Energy Research Center of Clausthal University of Technology 
EFZN) for help with both laser setups. We thank Philipp Hentrich and Andreas Schulz (Clausthal University of Technology, Institute of Inorganic and Analytical Chemistry) for supplying us with chlorine gas, Felix Lederle (Clausthal University of Technology, Institute of Technical Chemistry) for help with the UV/Vis/NIR spectra and Trotec Laser GmbH for nanosecondlaser structured aluminium plates.

\section{Notes and references}

1 A. Y. Vorobyev and C. Guo, Laser Photonics Rev., 2013, 7, 385407.

2 J. Lu, T. Huang, Z. Liu, X. Zhang and R. Xiao, Appl. Surf. Sci., 2018, 459, 257-262.

3 A.-M. Kietzig, S. G. Hatzikiriakos and P. Englezos, Langmuir, 2009, 25, 4821-4827.

4 C.-j. Yang, X.-s. Mei, Y.-l. Tian, D.-w. Zhang, Y. Li and X.-p. Liu, Int. J. Adv. Manuf. Technol., 2016, 87, 1663-1670.

5 A. Cunha, A. P. Serro, V. Oliveira, A. Almeida, R. Vilar and M.-C. Durrieu, Appl. Surf. Sci., 2013, 265, 688-696.

6 A. Y. Vorobyev and C. Guo, Proc. SPIE, 2008, 7005, 70051T.

7 A. Y. Vorobyev and C. Guo, Adv. Mech. Eng., 2010, 2010, 452749.

8 R. Younkin, J. E. Carey, E. Mazur, J. A. Levinson and C. M. Friend, J. Appl. Phys., 2003, 93, 2626-2629.

9 C. H. Crouch, J. E. Carey, J. M. Warrender, M. J. Aziz, E. Mazur and F. Y. Génin, Appl. Phys. Lett., 2004, 84, 18501852.

10 J. Yang, Y. Yang, B. Zhao, Y. Wang and X. Zhu, Appl. Phys. B, 2012, 106, 349-355.

11 A. Y. Vorobyev, V. S. Makin and C. Guo, Phys. Rev. Lett., 2009, 102, 234301.

12 J. Heitz, C. Plamadeala, M. Muck, O. Armbruster, W. Baumgartner, A. Weth, C. Steinwender, H. Blessberger, J. Kellermair, S. V. Kirner, J. Krüger, J. Bonse, A. S. Guntner and A. W. Hassel, Appl. Phys. A, 2017, 123, 734.

13 E. Fadeeva, V. K. Truong, M. Stiesch, B. N. Chichkov, R. J. Crawford, J. Wang and E. P. Ivanova, Langmuir, 2011, 27, 3012-3019.

14 E. Fadeeva, S. Schlie, J. Koch and B. N. Chichkov, J. Adhes. Sci. Technol., 2010, 24, 2257-2270.

15 A. Y. Vorobyev and C. Guo, Appl. Surf. Sci., 2007, 253, 72727280.

16 S. Shaikh, S. Kedia, D. Singh, M. Subramanian and S. Sinha, J. Laser Appl., 2019, 31, 022011.

17 S. B. Beil, T. Müller, S. B. Sillart, P. Franzmann, A. Bomm, M. Holtkamp, U. Karst, W. Schade and S. R. Waldvogel, Angew. Chem., Int. Ed., 2018, 57, 2450-2454.

18 P. Faubert, C. Müller, H. Reinecke, P. Smyrek, J. Proell and W. Pfleging, MRS Proceedings, 2015, 1777, 7-13.

19 A. Gabler, C. I. Müller, T. Rauscher, T. Gimpel, R. Hahn, M. Köhring, B. Kieback, L. Röntzsch and W. Schade, Int. J. Hydrogen Energy, 2018, 43, 7216-7226.

20 A. R. Neale, Y. Jin, J. Ouyang, S. Hughes, D. Hesp, V. Dhanak, G. Dearden, S. Edwardson and L. J. Hardwick, J. Power Sources, 2014, 271, 42-47.
21 K. M. T. Ahmmed, C. Grambow and A.-M. Kietzig, Micromachines, 2014, 5, 1219-1253.

22 B. K. Nayak, M. C. Gupta and K. W. Kolasinski, Appl. Phys. A, 2008, 90, 399-402.

23 C. S. R. Nathala, A. Ajami, W. Husinsky, B. Farooq, S. I. Kudryashov, A. Daskalova, I. Bliznakova and A. Assion, Appl. Phys. A, 2016, 122, 107.

24 K.-H. Leitz, B. Redlingshöfer, Y. Reg, A. Otto and M. Schmidt, Phys. Procedia, 2011, 12, 230-238.

25 H. O. Jeschke, M. E. Garcia, M. Lenzner, J. Bonse, J. Krüger and W. Kautek, Appl. Surf. Sci., 2002, 197-198, 839-844.

26 M. Hashida, A. F. Semerok, O. Gobert, G. Petite, Y. Izawa and J. F. Wagner, Appl. Surf. Sci., 2002, 197-198, 862-867.

27 B. K. Nayak and M. C. Gupta, Opt. Lasers Eng., 2010, 48, 940949.

28 B. K. Nayak and M. C. Gupta, Opt. Lasers Eng., 2010, 48, 966973.

29 V. Oliveira, A. Cunha and R. Vilar, J. Optoelectron. Adv. Mater., 2010, 12, 654-658.

30 V. Oliveira, S. Ausset and R. Vilar, Appl. Surf. Sci., 2009, 255, 7556-7560.

31 M. Tsukamoto, K. Asuka, H. Nakano, M. Hashida, M. Katto, N. Abe and M. Fujita, Vacuum, 2006, 80, 1346-1350.

32 J. Lehr, F. de Marchi, L. Matus, J. MacLeod, F. Rosei and A.-M. Kietzig, Appl. Surf. Sci., 2014, 320, 455-465.

33 J. Haubrich, K. Schulze, and J. Hausmann, Laser treatments of titanium surfaces In different atmospheres for Improved adhesion properties, Euro Hybrid Materials and Structures, 2014.

34 P. Mannion, J. Magee, E. Coyne and G. M. O'Connor, Proc. SPIE, 2003, 4876, 470-478.

35 J. Güdde, J. Hohlfeld, J. G. Müller and E. Matthias, Appl. Surf. Sci., 1998, 127-129, 40-45.

36 S. Biswas, A. Karthikeyan and A.-M. Kietzig, Materials, 2016, 9, 1023.

37 Communications: Laser Technik Journal, Laser Tech. J., 2015, 12, 3, 22-26, DOI: 10.1002/latj.201590031.

38 CRC Handbook of Chemistry and Physics, ed. D. R. Lide, CRC Press LLC, Boca Raton, 84th edn, 2004.

39 Gmelin Handbook of Inorganic Chemistry - Titan, ed. R. J. Meyer and E. H. E. Pietsch, Springer Verlag, Berlin, 8th edn, 1951.

40 L. T. Zhuravlev, Colloids Surf., A, 2000, 173, 1-38.

41 M. Trtica, D. Batani, R. Redaelli, J. Limpouch, V. Kmetik, J. Ciganovic, J. Stasic, B. Gakovic and M. Momcilovic, Laser Part. Beams, 2013, 31, 29-36.

42 N. H. Nordin and Z. Ahmad, J. Phys. Sci., 2015, 26, 11-22.

43 J. J. Cras, C. A. Rowe-Taitt, D. A. Nivens and F. S. Ligler, Biosens. Bioelectron., 1999, 14, 683-688.

44 H. G. Hecht, J. Res. Natl. Bur. Stand., Sect. A, 1976, 80, 567583.

45 N. G. Zubrilin, I. V. Blonskiy, I. M. Dmitruk, O. E. Domrovskiy, N. I. Berezovska and V. I. Stiopkin, Semicond. Phys., Quantum Electron. Optoelectron., 2017, 20, 48-54.

46 I. Gnilitskyi, T. J.-Y. Derrien, Y. Levy, N. M. Bulgakova, T. Mocek and L. Orazi, Sci. Rep., 2017, 7, 8485. 
47 S. Höhm, A. Rosenfeld, J. Krüger and J. Bonse, Opt. Express, 2015, 23, 25959-25971.

48 C. Zwahr, R. Helbig, C. Werner and A. F. Lasagni, Sci. Rep., 2019, 9, 6721.

49 W. B. Chou, M. N. Azer and J. Mazumder, J. Appl. Phys., 1989, 66, 191-195.
50 Y. Yang, J. Yang, C. Liang, H. Wang, X. Zhu and N. Zhang, Opt. Express, 2009, 17, 21124-21133.

51 Y. Liu, L. Tian, X. Tan, X. Li and X. Chen, Sci. Bull., 2017, 62, 431-441. 